\title{
EMITTANCE MEASUREMENTS AT THE TTF PHOTOINJECTOR
}

\author{
Ph. Piot, S. Schreiber ${ }^{*}$, D. Sertore ${ }^{\dagger}$, K. Flöttmann, DESY, 22603 Hamburg, Germany, \\ A. Cianchi, INFN Frascati, Italy, L. Catani, INFN Roma II, Italy.
}

\begin{abstract}
The TTF Free Electron Laser requires high current electron bunches with small emittances. The beam is produced by an injector based on a laser driven rf gun. The design aims for a bunch charge of $1 \mathrm{nC}$, a bunch length in the $\mathrm{mm}$ range, and a normalized transverse emittance approaching $1 \mathrm{~mm}$ mrad. Emittance measurements in the photoinjector after preacceleration to $17 \mathrm{MeV}$ are presented and compared to simulations.
\end{abstract}

\section{INTRODUCTION}

The TESLA Test Facility (TTF) operates an rf gun based photoinjector.[1] Various experiments related to the TESLA linear collider project require electron bunches with high peak current, but at the same time with a small transverse emittance in both planes while keeping the energy spread small. Excellent beam properties are essential to drive the TTF-FEL free electron laser [2]. Besides TTF, two other test facilities, one based at Fermilab [3], one based at DESY Zeuthen [4] serve as test beds to optimize rf gun based injectors for next generation linear accelerator projects. At Fermilab, an extensive study of the emittance has already been carried out [5], Zeuthen plans to have their first beam late summer this year.

This report, cannot give a complete discussion on the emittance measured at TTF, but tries to show, that the measurements performed so far are widely understood in terms of simulations, but that the optimization of the emittance for the given design has not yet been completed. Experimental tools to measure and understand the transverse emittance are not yet satisfactory and hardly allow to scan the complete parameter space.

\section{EXPERIMENTAL SET-UP}

A schematic overview of the TTF injector is shown in Fig. 1, further details can be found in [1]. The electron source is a laser-driven L-band of gun with a $\mathrm{Cs}_{2} \mathrm{Te}$ cathode. The cathode is illuminated by a train of UV laser pulses generated in a mode-locked solid-state laser system synchronized with the gun rf. The gun section is followed by a booster consisting of a standard TESLA 9-cell superconducting accelerating cavity. The booster is operated at $12 \mathrm{MV} / \mathrm{m}$, the beam energy measured at the energy spectrometer is $16.5 \mathrm{MeV}$. For this experiment, the bunch compressor installed downstreams of the booster has not been

\footnotetext{
* Email: siegfried.schreiber@ desy.de

${ }^{\dagger}$ now at INFN LASA, Milano, Italy
}

used. The summary of the injector design parameters are listed in Table 1.

Table 1: Injector design parameters for TESLA related experiments (TTFL) and TTF-FEL operation. The actual operating parameters mentioned in the text may differ from this table.

\begin{tabular}{l|c|c|c|c}
\hline Parameter & & \multicolumn{2}{|c|}{ TTFL } & FEL \\
\cline { 3 - 4 } & & (a) & (b) \\
\hline RF frequency & $\mathrm{GHz}$ & \multicolumn{3}{|c}{1.3} \\
Rep. rate & $\mathrm{Hz}$ & \multicolumn{3}{|c}{10} \\
Pulse train length & $\mu \mathrm{s}$ & \multicolumn{3}{|c}{800} \\
\cline { 3 - 5 } Pulse train current & $\mathrm{mA}$ & 8 & 9 & 9 \\
Bunch frequency & $\mathrm{MHz}$ & 1 & 2.25 & 9 \\
Bunch charge & $\mathrm{nC}$ & 8 & 4 & 1 \\
Bunch length (rms) & $\mathrm{mm}$ & 1 & 1 & 0.8 \\
Emitt. norm. (x,y) & $\mu \mathrm{m}$ & 20 & 10 & 2 \\
\cline { 3 - 5 }$\Delta \mathrm{E} / \mathrm{E}$ (rms) & $\%$ & \multicolumn{3}{|c}{0.1} \\
EE/E (bunch to bunch) (rms) & $\%$ & \multicolumn{3}{|c}{20.2} \\
Injection energy & $\mathrm{MeV}$ & \multicolumn{3}{|c}{20} \\
\hline
\end{tabular}

The emittance has been measured with two methods, the quadrupole scan, and the multislit mask technique.

In the first method, the beam size is measured as a function of the magnetic gradient of a quadrupole at the exit of the booster. The emittance is then calculated by fitting the data to the prediction given by beam transport equations. Space charge effects limit this method, but for the data presented here, these effects have been accounted for and are small but not negligible. The method has been validated by simulations.

The multislit mask technique cuts the beam into beamlets with reduced charge. Space charge effects are thus widely suppressed. The beamlets are allowed to drift a proper length to a screen. From the size and number of beamlets, the beam divergence and its size are calculated, yielding the emittance.

In both cases, an aluminum foil serves as radiator of optical transition radiation (OTR). The beam is imaged to a CCD camera for the quadrupole scans (OTR INJ5 in Fig. 1). and onto an intensified camera for the slit measurements (OTR INJ4 in Fig. 1).

The slits width is $50 \mu \mathrm{m} ; 10$ slits are mounted with a spacing of $1 \mathrm{~mm}, 10$ slits with $0.5 \mathrm{~mm}$. They are built from Tungsten bars with a thickness of $4 \mathrm{~mm}$. The slits are moved into the beam line with a special motorized actuator, which allows precise adjustment in height and in angle. 


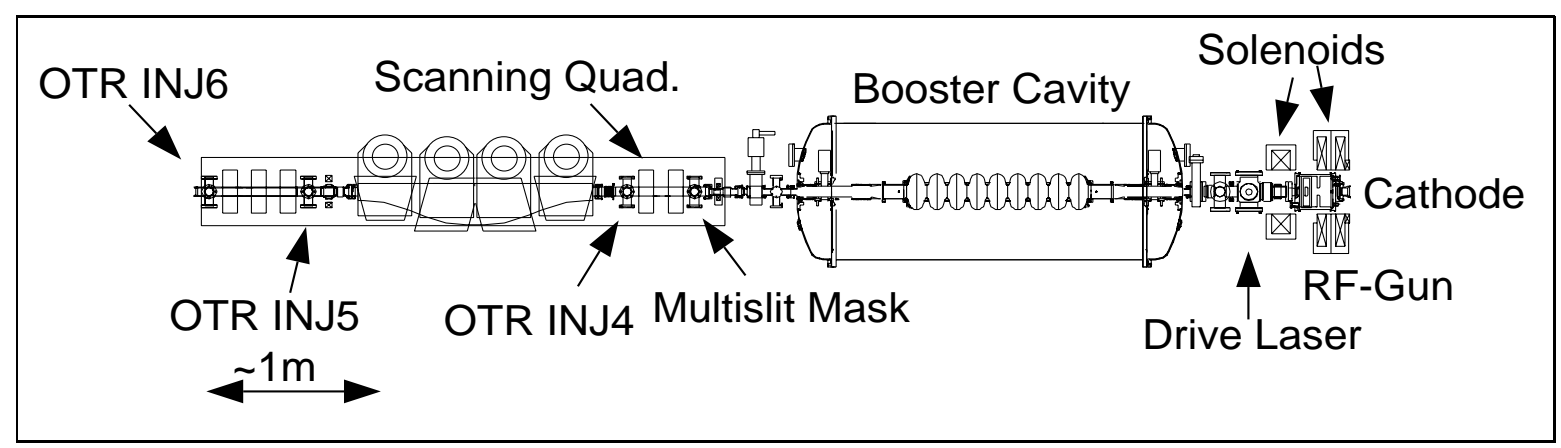

Figure 1: Schematic overview of the TTF injector. Beam direction is from right to left; the laser system is not shown. The quadrupole used for the emittance measurements as well as the multislit mask, and the OTR screens are indicated.

The slits are mounted directly at the exit of the booster cavity after acceleration to $16.5 \mathrm{MeV}$ (see Fig. 1). The screen is placed in $385 \mathrm{~mm}$ distance from the slits.

For this experiment, the laser spot size on the cathode has been kept constant to a radius of $1.5 \mathrm{~mm}$. The charge per bunch is set to $1 \mathrm{nC}$. The laser pulse length is $7 \mathrm{pm}$ $1 \mathrm{ps}$, the energy spread is measured to be $0.13 \pm 0.02 \%$. The relative phase of the gun rf to the laser is chosen to be $40^{\circ}$ close to the optimum determined by simulations of the beam dynamic in the rf gun.

\section{THE MULTISLIT TECHNIQUE}

In the following, the analysis method used for the slit technique is briefly described. In general, the statistical emittance is defined as $\varepsilon_{y}=\sqrt{\sigma_{y}^{2} \cdot \sigma_{y^{\prime}}^{2}-\operatorname{cov}^{2}\left(y, y^{\prime}\right)}$. By shearing the phase space ellipse with the transformation $y \mapsto y, y^{\prime} \mapsto y^{\prime}-\kappa y$, which does not change the emittance value, the correlation term vanishes and the emittance is just given by the square root of the product of the variances in $y$ and $y^{\prime}$. After removing occasional $\mathrm{X}$-rays from that recorded images by a software filter, the beamlets are projected on the vertical axis. In most of the measurements, three to five beamlets have been seen. Under certain conditions, some of the beamlets may overlap partially. Therefore, all beamlets are fitted together to a distribution which consists of several gaussian functions with a constant background. From the fit, the position and width of each beamlet is determined and used to calculate the emittance.

\section{MEASUREMENTS AND RESULTS}

First, the emittance is measured as a function of the relative phase between the laser and the rf of the gun. Figure 2 shows the measured emittance, spot size, and beam divergence with both methods, the slit and the quadrupole technique. Simulations with the code ASTRA[6] are shown as well. The simulation follows well the data for both methods. The dependence of the emittance on the phase is small as expected. The quadrupole scan data are closer to the prediction, while the slit data give larger emittances but with larger measurement errors. The solenoid fields have been
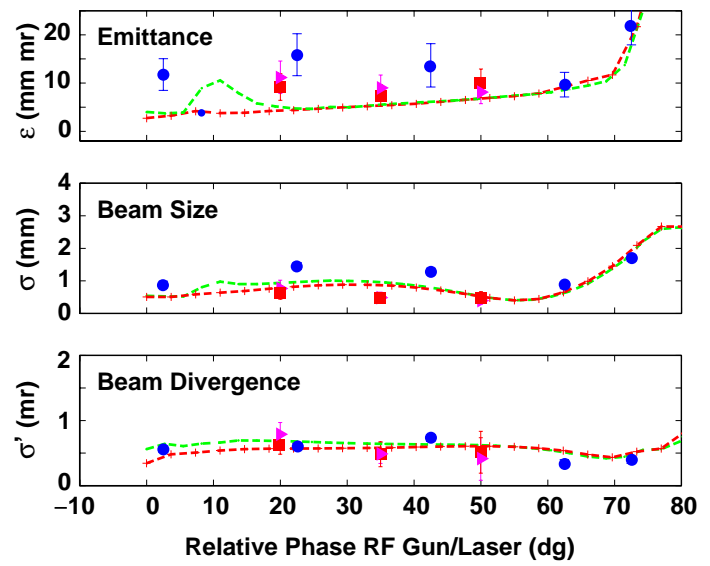

Figure 2: Transverse emittance, spot size, and divergence as a function of the relative phase $\mathrm{rf}$ gun/laser. Squares (vert.) and triangles (hor.) show the results obtained by the quadrupole scan method, dots the data from the slits technique (vert. only). The dashed line is a simulation with ASTRA.

kept constant for these measurements.

In a next series, the emittance is measured as a function of solenoid settings. The emittance compensation technique [7] is based on a solenoidal field at the gun which if proper set - compensates the space charge induced emittance growth. Figure 3 shows the measurements with the slit method for different combination of currents in both solenoids. The simulations follow nicely the data points. However, measurements have not been possible for high solenoid fields, where the emittance is predicted to increase strongly with the solenoid field.

A scan of the solenoids has also been performed using quadrupole scans. The smallest emittance measured for a solenoid field of $0.105 \mathrm{~T}$ for the first and $0.088 \mathrm{~T}$ for the second solenoid is $3.0 \pm 0.2 \mathrm{~mm}$ mrad (Fig. 4). 


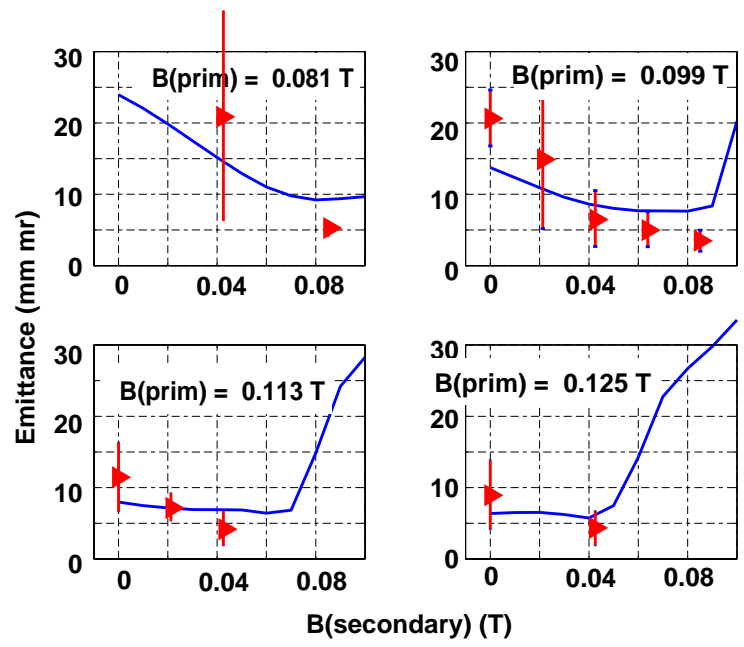

Figure 3: Vertical emittance measured with the slit technique as a function of field of the second solenoid. The four plots are for different fields of the first solenoid. The line shows a simulation with ASTRA.

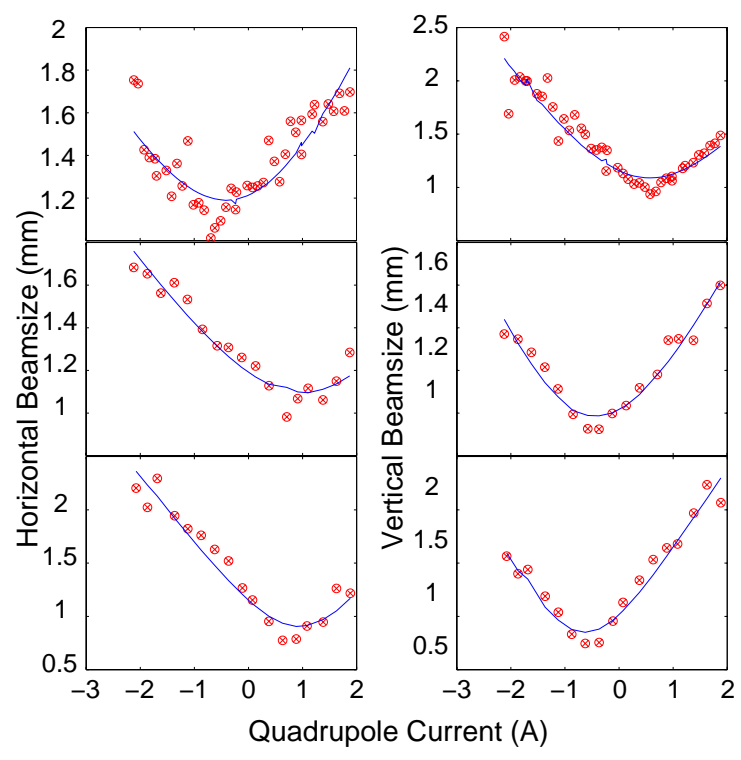

Figure 4: Example of a quadrupole scan for three different solenoid fields: Solenoid $1=0.099 \mathrm{~T}$ (upper), $0.105 \mathrm{~T}$ (middle), and $0.110 \mathrm{~T}$. Solenoid 2 is constant at $0.088 \mathrm{~T}$. The measured beam sizes is plotted as a function the of quadrupole current. A fit to the data is indicated. The fit to the middle row data gives an emittance of $3.0 \pm 0.2$ $\mathrm{mm} \mathrm{mrad}$ (hor.) and $3.5 \pm 0.1$ (vert.).

\section{DISCUSSION AND CONCLUSION}

The data are in a quite good agreement with the simulations. Therefore, we believe to understand widely the beam dynamics in the TTF photoinjector. On the other hand, the finding of an optimized parameter set is very difficult and time consuming, and has not yet been finalized yet. However, the present settings have already a reasonable performance, even if the goal of 1 to $2 \mathrm{~mm}$ mrad for $1 \mathrm{nC}$ has not been reached so far.

A very complete and extensive study of the emittance at the Fermilab/NICCAD photoinjector test facility at A0 has shown, that there are still inconsistencies between the data and the models as well as between the models themselves.[5] Further measurements are scheduled to improve the parameter sets and to study possible new injector designs.

\section{REFERENCES}

[1] S. Schreiber, "Performance Status of the RF-gun based injector of the TESLA Test Facility Linac", Proc. of the Europ. Particle Acc. Conf. EPAC2000, Vienna, Austria, p. 309.

[2] J. Andruszkow et al., "First Observation of Self-Amplified Spontaneous Emission in Free-Electron Laser at $109 \mathrm{~nm}$ Wavelength”, Phys. Rev. Lett. 85 (2000) 3825-3829.

[3] J.-P. Carneiro et al., "First Results of the Fermilab HighBrightness RF Photoinjector", Proc. of the 1999 Particle Accelerator Conference, New York, 1999, p. 2027.

[4] F. Stephan et al, "Photo Injector Test Facility under Construction at DESY Zeuthen”, Proc. 22nd Free Electron Laser Conf., 13-18 Aug 2000, Durham, North Carolina, USA, to be published.

[5] J.-P. Carneiro, "Etude expérimentale du photo-injecteur de Fermilab", PhD-Thesis, Univ. de Paris-Sud, Orsay, France.

[6] K. Flöttmann, ASTRA user manual, ww. desy.de/ ${ }^{\sim}$ mpyflo/ASTRA_dokumentation .

[7] B. E. Carlsten et al., Nucl. Instr. and Meth. A285 (1989) 313. 